\title{
Experiment-based modelling of the mechanical behaviour of non- hazardous waste incineration bottom ashes treated by hydraulic binder
}

\section{Approche couplée modélisation-expérimentation pour étudier le comportement mécanique de Mâchefers d'Incinération de Déchets Non Dangereux traités avec un liant hydraulique}

\author{
Libasse Sow $^{1,2}$, Fabrice Bernard ${ }^{2}$, Siham Kamali-Bernard ${ }^{2}$, Cheikh Mouhamed Fadel Kébé ${ }^{1}$ \\ ${ }^{1}$ Ecole Supérieure Polytechnique, Université Cheikh Anta Diop de Dakar, Sénégal \\ ${ }^{2}$ Laboratoire de Génie Civil et Génie Mécanique (LGCGM) INSA Rennes, France
}

\begin{abstract}
Instrumented indentation tests have been carried out on an isolated $25 \mathrm{~mm}$ diameter particle of Non-Hazardous Waste Incineration bottom ash. These tests have enabled one to assess the intrinsic mean reduced modulus of elasticity " $E_{r}$ " of the particles. This result is used as input data for a 3D numerical model of Representative Elementary Volumes (REV) of a road gravel made with this kind of by-products. This numerical model is based on a multi-scale hierarchical modelling strategy. The aggregates treated with cement have been decomposed into two REV at the sub-mesoscopic and mesoscopic scales. The numerical simulations campaign ("virtual laboratory") lead to the following results. At the sub-mesoscopic scale, we determined the input parameters for the Concrete Damaged Plasticity Model (CDPM) used at the mesoscopic scale. At the mesoscopic scale, the mechanical characteristics of the road aggregates usually determined through experiments have been found. The non-hazardous waste incineration bottom ashes treated by hydraulic binder was classified into mechanical classe 3 .
\end{abstract}

\begin{abstract}
Résumé. Des tests d'indentation instrumentée ont été effectués sur particule isolée de Mâchefers d'Incinération de Déchets Non Dangereux (MIDND) de diamètre $25 \mathrm{~mm}$. Ces tests ont permis d'obtenir une estimation du module élastique réduit moyen « $E_{\mathrm{r}}$ » intrinsèque aux particules étudiées. Ce résultat sert de donnée d'entrée à une modélisation numérique tridimensionnelle de Volumes Elémentaires Représentatifs (VER) d'une grave routière à base de ces MIDND. Le modèle numérique est basé sur une stratégie de modélisation multi-échelle hiérarchique. Les granulats traités au ciment ont été décomposés en deux VER : sub-mesoscopique et mesoscopique. A l'échelle sub-mesoscopique, nous avons déterminé les paramètres permettant d'alimenter le modèle d'endommagement plastique (CDPM) utilisé à l'échelle mesoscopique. A l'échelle mesoscopique, les caractéristiques mécaniques de la grave routière traitée au ciment, habituellement déterminées par le biais d'expérimentations, ont été retrouvées. La grave routière à base de MIDND traitée au ciment a été classée en classe mécanique 3 .
\end{abstract}

\section{Introduction}

La gestion des déchets est un enjeu majeur face à l'augmentation incessante de la quantité produite quotidiennement. Cependant, il est possible de les valoriser sous forme de Mâchefers d'Incinération de Déchets Non Dangereux (MIDND). Les mâchefers répondant aux exigences de la règlementation en vigueur [1] peuvent présenter des caractéristiques permettant leur valorisation en techniques routières. Il n'existe à ce jour aucune connaissance précise sur les caractéristiques élastiques intrinsèques de ces types de matériaux. Par ailleurs, l'approfondissement des connaissances et surtout le développement des matériaux cimentaires à base de produits recyclés ont montré que la structure du matériau était beaucoup trop complexe et aléatoire pour permettre le développement de formules analytiques exactes qui décrivent comment les propriétés et les performances lui sont reliées. Parallèlement à ce constat, le développement incessant des possibilités de calcul numérique et des moyens informatiques a permis le développement de simulations basées sur une description explicite de plus en plus réaliste des matériaux cimentaires. Ainsi, depuis quelques années, le «calcul des microstructures » et les méthodes de changement d'échelles se sont fortement développés dans la communauté mécanicienne au sens large du terme. Dans ce travail, une démarche de modélisation hiérarchique est mise en place à l'échelle mesoscopique afin d'étudier le comportement mécanique d'une grave routière à base de MIDND traitée avec un 
liant hydraulique. Cette démarche de modélisation nécessite d'être alimentée par des données expérimentales permettant de caractériser les propriétés élastiques intrinsèques des particules de MIDND valorisées. Ces propriétés sont obtenues dans ce travail par le biais d'une campagne d'indentation originale et jamais menée jusqu'à maintenant sur des granulats de mâchefers. L'indentation permet l'obtention du module élastique réduit en utilisant la méthode décrite dans [2].

\section{Résultats d'une campagne d'indentation instrumentée sur particule isolée de mâchefer}

\subsection{Matériaux étudiés}

Les mâchefers étudiés proviennent des carrières de la Garenne à Vignoc en Bretagne (France), ils sont sur la plateforme de maturation depuis environ une année et demie. Les granulats de mâchefers étudiés sont présentés à la figure 1.a avec la fraction 12,5/25 mm. Un exemple de particule isolée de mâchefer de diamètre $25 \mathrm{~mm}$, d'apparence "amorphe » découpée en sa ligne médiane et moulée dans de la résine est montrée à la figure 1.b. Les tests d'indentation ont été effectués sur cette particule isolée.

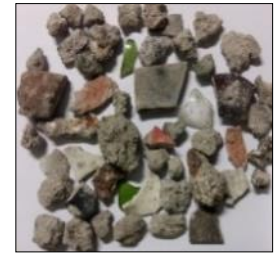

(a)

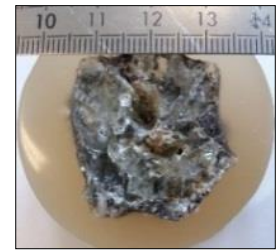

(b)
Fig.1. Echantillons de mâchefers étudiés

\subsection{Détermination des propriétés élastiques intrinsèques des particules de MIDND}

Deux séries d'indentation ont été réalisées avec un indenteur sphérique de rayon $140 \mathrm{~mm}$ en carbure de tungstène dont les caractéristiques élastiques sont connues. La procédure d'essais est plus précisément décrite dans [3]; les essais permettent de suivre l'évolution de l'effort appliqué en fonction de l'enfoncement réel de l'indenteur. Avec un indenteur de $140 \mathrm{~mm}$ de rayon, un plus grand volume de matériau est testé. Il intègre les différentes phases solides mais aussi la porosité et donne des modules homogénéisés de la particule testée. La déformation du porte-indenteur (correction de la compliance du banc expérimental) est prise en compte. La phase de décharge est considérée suivre la relation puissance de l'équation (1).

$P=A^{*}\left(h-h_{f}\right)^{m}$

La figure 2 montre un exemple de courbe effortenfoncement obtenue. A partir des courbes d'indentation obtenues, ont été déterminés les modules élastiques réduits caractérisant les mâchefers étudiés (Tableau 1). Le module réduit d'un matériau s'exprime en fonction de son module d'Young et son coefficient de Poisson ; il est en principe impossible de découpler l'un de l'autre. Toutefois, en considérant la gamme complète de valeurs que peut prendre le coefficient de Poisson, il est possible de déduire que le module d'Young homogénéisé des particules de MIDND est compris entre 15 et $20 \mathrm{GPa}$.

Tableau 1. Modules élastiques réduits obtenus

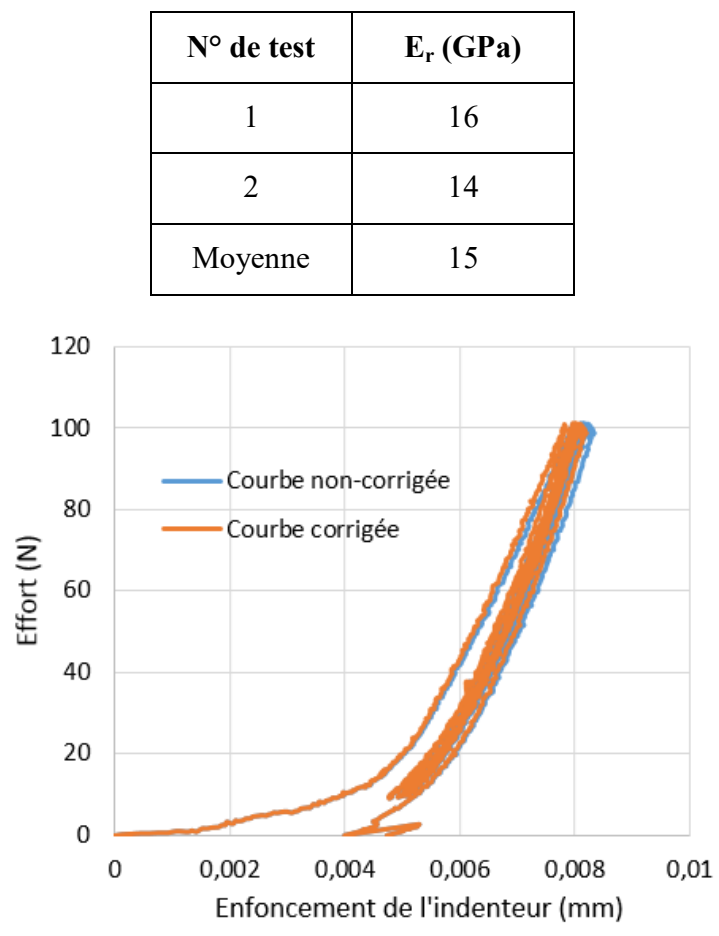

Fig.2. Exemple de courbe d'indentation -Test 1

\section{Modélisation numérique hiérarchique multi-échelle tridimensionnelle}

\subsection{Stratégie spécifique de modélisation}

La fraction granulométrique des particules de MIDND $(0 / 25 \mathrm{~mm})$ est trop étendue pour être considérée dans son intégralité dans une modélisation aux éléments finis 3D. Deux échelles d'étude ont donc été considérées : l'échelle sub-mesoscopique contenant la fraction $0 / 6 \mathrm{~mm} \mathrm{du}$ squelette granulaire noyée dans une matrice poreuse de liant hydraulique (ou micro-grave-ciment, MGC) et l'échelle mesoscopique contenant la fraction $6 / 25 \mathrm{~mm}$, noyée dans la MGC. A l'échelle mesoscopique, un modèle d'endommagement plastique (CDPM) est retenu pour caractériser la micro-grave-ciment. Ce modèle utilise une variable scalaire d'endommagement, la surface de charge de Lee and Fenves [4] et le potentiel plastique de Drucker-Prager (version hyperbolique). Il requiert la détermination de paramètres par le biais de simulations menées à l'échelle sub-mesoscopique. Le module d'Young (E), le coefficient de Poisson (v), la résistance en compression uniaxiale $\left(\mathrm{f}_{\mathrm{b}}\right)$ sont déterminés à partir des 
tests uniaxiaux de compression. La résistance en compression biaxiale $\left(\mathrm{f}_{\mathrm{b} 0}\right)$ est obtenue avec le test de compression biaxiale. Le degré de déformabilité du matériau $(\mathrm{K})$, le coefficient $\mathrm{M}_{\text {pic }}$ (rapport entre le déviateur des contraintes au pic et la contrainte moyenne), l'angle de frottement interne $(\varphi)$ et l'angle de dilatance $(\psi)$ sont obtenus par le biais de tests triaxiaux. Les figures 3, 4, 5 et 6 décrivent respectivement la compression uniaxiale, la compression biaxiale, la traction uniaxiale et le test de triaxial de compression.

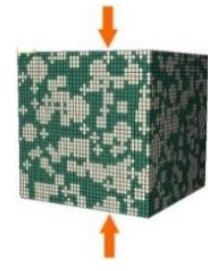

Fig.3. Compression uniaxiale

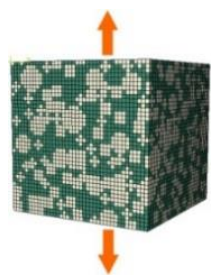

Fig.5. Traction uniaxiale

Fig.6. Triaxial de compression

La stratégie de modélisation adoptée est résumée dans la figure 7 .

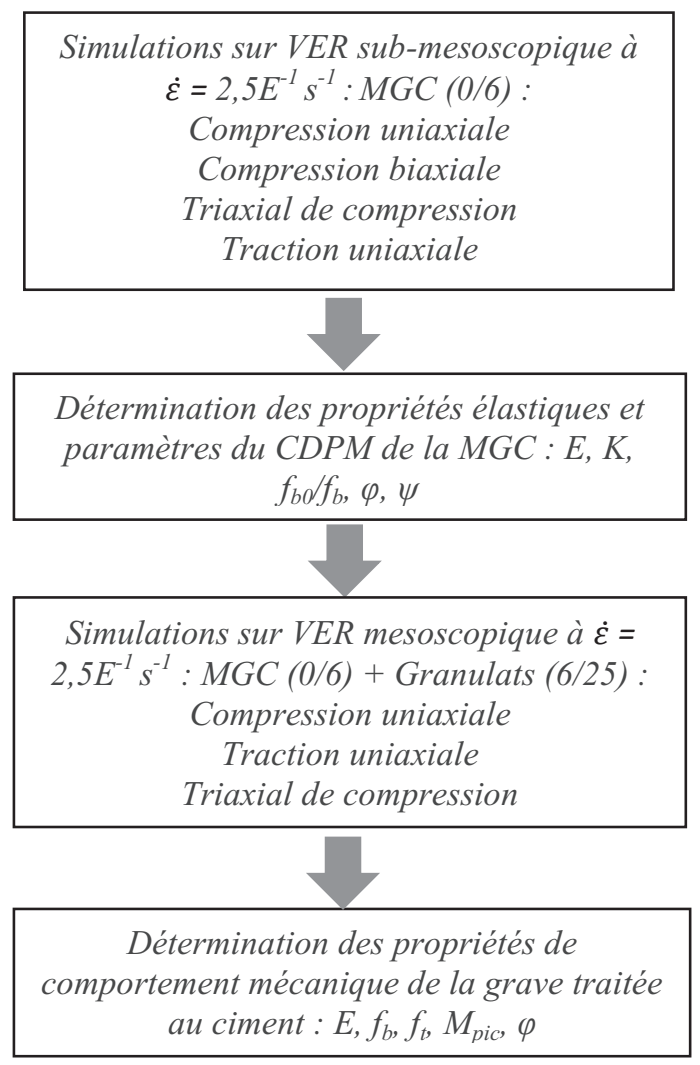

Fig.7. Stratégie de modélisation hiérarchique développée dans ce travail
Avec un test triaxial de compression, il est possible de tracer les courbes déviatoriques et de déformations volumiques ainsi que la réponse du matériau dans le plan $(\mathrm{q}, \mathrm{p})$, avec $\mathrm{q}$ le déviateur des contraintes et $\mathrm{p}$ la contrainte moyenne. A partir de ces courbes, l'angle de dilatance $(\psi)$ et l'angle de frottement interne $(\varphi)$ sont identifiés par l'intermédiaire des équations (2) et (3).

$\Psi=\arcsin \left(\frac{\frac{d \varepsilon_{v}}{d \varepsilon_{1}}}{2+d \varepsilon_{v} / d \varepsilon_{1}}\right)$
$\varphi=\arcsin \left(\frac{3 M}{M+6}\right)$

M : rapport entre le déviateur de contraintes (q) et la contrainte moyenne (p).

Selon [5], le paramètre $\mathrm{K}$ caractérisant le degré de déformabilité du matériau peut être déterminé par la relation de l'équation (4). Le paramètre $\alpha$ se détermine quant à lui par la relation de l'équation (5).

$K=\frac{(1-\alpha)(\sin \varphi-3)}{2 \alpha(3-\sin \varphi)-(\sin \varphi+3)}$
$\alpha=\frac{1-A}{-2 A+1}$

$\mathrm{A}=\mathrm{f}_{\mathrm{b} 0} / \mathrm{f}_{\mathrm{b}}$ : rapport entre la résistance en compression biaxiale et la résistance en compression uniaxiale.

\subsection{Construction des Volumes Elémentaires Représentatifs (VER)}

Afin de modéliser la granulométrie étalée $0 / 25 \mathrm{~mm}$ avec la présence d'une pâte de ciment comme liant, il est nécessaire de représenter l'hétérogénéité avec les différentes phases explicites qui la caractérisent : granulats, liant, porosité. Les volumes élémentaires représentatifs aux deux échelles suivent les compositions données aux tableaux 2 et 3 .

Tableau 2. Composition du VER sub-mesoscopique

\begin{tabular}{|c|c|c|c|}
\hline \multirow{2}{*}{ Matrice : 15\% } & \multicolumn{3}{|c|}{$\begin{array}{c}\text { Squelette granulaire : } \\
\text { micro-grave 0/6 à 85\% }\end{array}$} \\
\hline $\begin{array}{c}\text { Pâte de ciment } \\
42,5 \mathrm{R}\end{array}$ & $\begin{array}{c}40 \%, \\
\Phi=1\end{array}$ & $\begin{array}{c}31 \%, \\
\Phi=3\end{array}$ & $\begin{array}{c}14 \%, \\
\Phi=5\end{array}$ \\
\hline
\end{tabular}

Tableau 3. Composition du VER mesoscopique

\begin{tabular}{|c|c|c|c|c|}
\hline $\begin{array}{c}\text { Matrice : } \\
\mathbf{4 7 , 5 \%}\end{array}$ & \multicolumn{4}{|c|}{ Squelette granulaire : grave 6/25 à 52,5\% } \\
\hline MGC & $22 \%$ de & $14,5 \%$ de & $11 \%$ de & $5 \%$ de \\
& $\Phi=7,5$ & $\Phi=12,5$ & $\Phi=17,5$ & $\Phi=22,5$ \\
\hline
\end{tabular}

$\Phi$ : diamètre moyen des granulats en $\mathrm{mm}$

La génération de pourcentages de grains aussi grands étant difficile, une méthode du «Take and place» sur un maillage Eléments Finis régulier existant a été utilisée afin de distribuer les grains à l'intérieur des VER. Les particules les plus petites de la MGC sont choisies 
cubiques pour permettre d'atteindre les pourcentages visés. Les structures obtenues ont ensuite été reprises dans Abaqus pour y effectuer les simulations. A l'échelle sub-mesoscopique, le VER est un cube $(40 * 40 * 40) \mathrm{mm}^{3}$ avec une résolution de $1 \mathrm{~mm}$. A l'échelle mesoscopique, la résolution est de $2,5 \mathrm{~mm}$ avec un VER cubique (100*100*100) $\mathrm{mm}^{3}$. Aux deux échelles, les éléments finis sont cubiques linéaires ; une étude de sensibilité sur la finesse du maillage a été menée.

\subsection{Caractéristiques mécaniques des matériaux}

\subsubsection{La pâte de ciment}

Le comportement post-pic de la pâte de ciment, donné par l'évolution bilinéaire de la contrainte post-pic en fonction de l'ouverture de fissure, est déterminé en considérant les résultats de [6]. Un modèle de fissuration diffuse, dont les paramètres sont déterminés par les relations 6 à 10, est alors utilisé.

$$
\begin{aligned}
& f_{1} / f_{0}=\left\{\begin{array}{l}
0,119 f_{\text {cap }-p}+0,8481 \\
-22,486 f_{\text {cap }-p}^{2}+15,99 f_{\text {cap }-p}-1,9525 \text { si } f_{\text {cap }-p} \leq 0,35
\end{array}\right. \\
& f_{0}=1,6673\left(1-f_{\text {cap }-p}\right)^{1,967}(\mathrm{MPa}) \\
& w_{1}=0,0661 f_{\text {cap }-p}^{-0,7565}(\mu \mathrm{m}) \\
& w_{2}=17,102 f_{\text {cap }-p}^{1,483}(\mu \mathrm{Hm}) \\
& E=35,931\left(1-f_{\text {cap }-p}\right)^{1,6973}(\mathrm{GPa})
\end{aligned}
$$

Avec : $\mathrm{f}_{0}$ : résistance en traction $; \mathrm{f}_{1}$ : contrainte à un point caractéristique de la forme bilinéaire; $\mathrm{w}_{1}$ et $\mathrm{w}_{2}$ : ouvertures de fissures correspondant à $\mathrm{f}_{1}$ et lorsque la résistance est nulle ; $\mathrm{f}_{\text {cap-p }}$ : porosité de la pâte de ciment.

Pour un temps d'hydratation long $(\alpha=0,83)$ correspondant à une porosité dans la matrice de $70 \%$, les propriétés mécaniques données au tableau 4 ont été obtenues.

Tableau 4. Valeurs caractéristiques de la forme bilinéaire postpic

\begin{tabular}{|c|c|c|c|c|c|}
\hline $\begin{array}{c}\mathbf{E} \\
(\mathbf{G P a})\end{array}$ & $\begin{array}{c}\mathbf{f}_{\mathbf{0}} \\
(\mathbf{M P a})\end{array}$ & $\begin{array}{c}\mathbf{f}_{\mathbf{1}} \\
(\mathbf{M P a})\end{array}$ & $\mathbf{f}_{\mathbf{1}} / \mathbf{f}_{\mathbf{0}}$ & $\begin{array}{c}\mathbf{w}_{\mathbf{1}} \\
(\boldsymbol{\mu} \mathbf{m})\end{array}$ & $\begin{array}{c}\mathbf{w}_{\mathbf{2}} \\
(\boldsymbol{\mu} \mathbf{m})\end{array}$ \\
\hline 4,17 & 0,138 & 0,138 & 0,91 & 0,09 & 10,45 \\
\hline
\end{tabular}

\subsubsection{Les granulats de mâchefers}

En raison de l'étendue de la granulométrie, les grains les plus petits (1 et $3 \mathrm{~mm}$ ) vont percoler d'une face à l'autre du VER. Afin d'empêcher que ce chemin continu ne modifie les résultats, un comportement fragile et donc une possible rupture de cette phase continue ont été imposés. La résistance de cette phase continue a été déterminée par une modélisation simplifiée permettant d'obtenir le comportement de granulats connectés équivalents à des granulats non-connectés. Ce comportement est caractérisé par le déplacement post-pic maximum $\left(\mathrm{u}_{\mathrm{ck}}\right)$, donné dans le tableau 5 pour les différentes sollicitations. Les autres granulats de diamètres $5 \mathrm{~mm}$ et plus sont considérés élastiques avec un module d'Young de $20 \mathrm{GPa}$.

Tableau 5. Caractéristiques des grains de diamètre 1 et $3 \mathrm{~mm}$

\begin{tabular}{|c|c|c|c|c|}
\hline Tests & $\begin{array}{c}\text { Compr. } \\
\text { biaxiale }\end{array}$ & $\begin{array}{c}\text { Compr. } \\
\text { Uniaxiale }\end{array}$ & $\begin{array}{c}\text { Triaxial } \\
\text { de compr. }\end{array}$ & $\begin{array}{c}\text { Traction } \\
\text { uniaxiale }\end{array}$ \\
\hline $\begin{array}{c}\mathbf{u}_{\mathbf{c k}} \\
(\mathbf{m m})\end{array}$ & $3,5 \mathrm{E}^{-4}$ & & $2 \mathrm{E}^{-4}$ & \\
\hline $\begin{array}{c}\mathbf{E}_{\mathbf{h o m}} \\
\mathbf{( G P a}\end{array}$ & \multicolumn{2}{|c}{14} \\
\hline
\end{tabular}

\subsection{Résultats obtenus aux deux échelles d'étude}

A l'échelle sub-mesoscopique, les paramètres caractérisant le comportement mécanique de la micrograve-ciment ont été obtenus (tableau 6). A l'échelle de la grave routière, les courbes de comportement obtenues sont données aux figures 8, 9 et 10. Le tableau 7 donne les caractéristiques mécaniques de la grave de MIDND traitée à $3 \%$ de ciment.

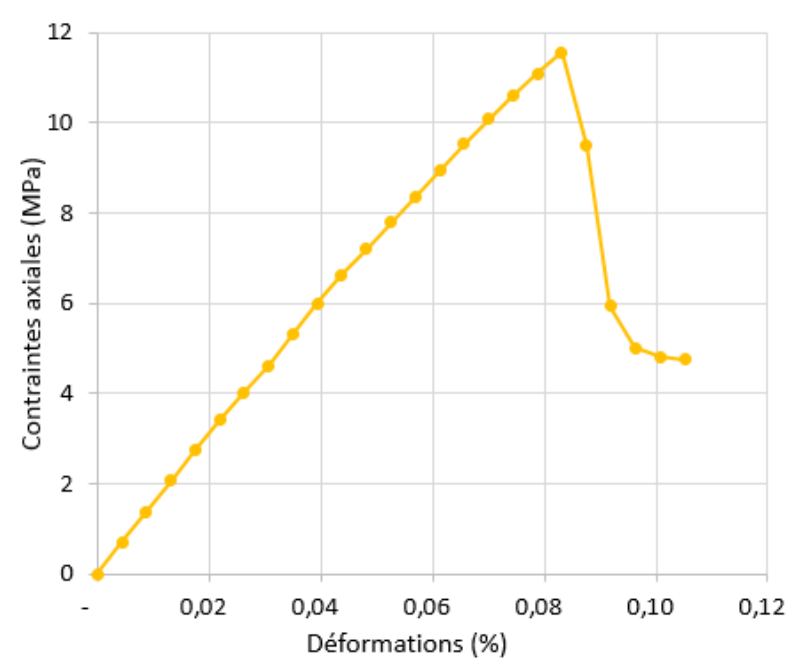

Fig.8. Courbe de comportement en compression uniaxiale

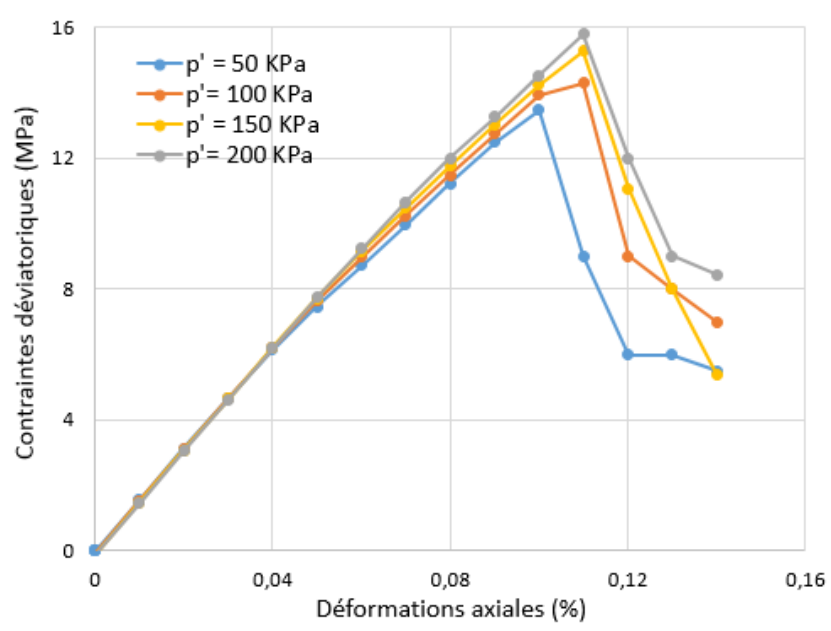

Fig.9. Courbes de comportement en triaxial de compression 


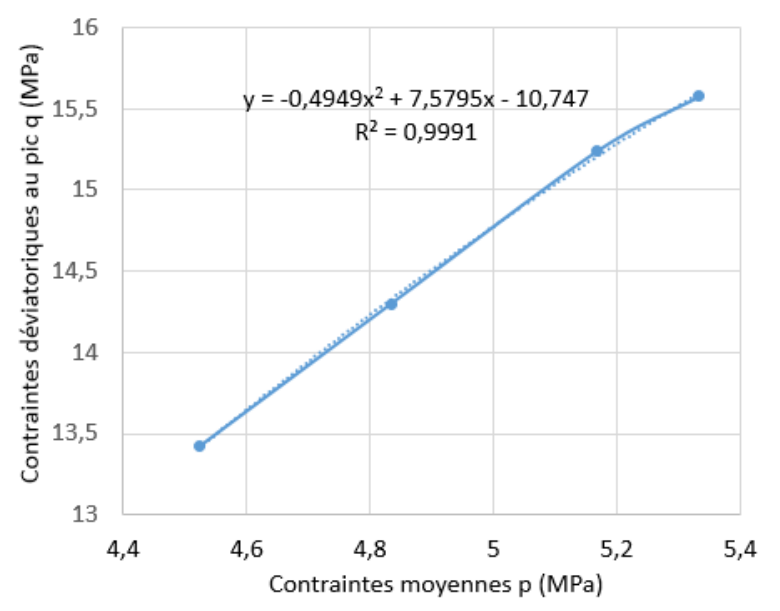

Fig.10. Résultats dans le plan (q, p)

Tableau 6. Paramètres du CDPM caractérisant la MGC

\begin{tabular}{|c|c|c|c|c|c|}
\hline $\begin{array}{c}\mathbf{E} \\
(\mathbf{G P a})\end{array}$ & $\mathbf{K}$ & $\mathbf{f}_{\mathbf{b} \mathbf{0}} / \mathbf{f}_{\mathbf{b}}$ & $\begin{array}{c}\boldsymbol{\psi} \\
\left({ }^{\circ} \mathbf{C}\right)\end{array}$ & $\mathbf{M}_{\text {pic }}$ & $\begin{array}{c}\boldsymbol{\varphi} \\
\left({ }^{\circ} \mathbf{C}\right)\end{array}$ \\
\hline 12,63 & 0,689 & 1,201 & 10 & 10,4 & 38 \\
\hline
\end{tabular}

Tableau 7. Caractéristiques mécaniques de la grave routière à base de MIDND traitée à $3 \%$ de ciment

\begin{tabular}{|c|c|c|c|c|}
\hline $\begin{array}{c}\mathbf{E} \\
(\mathbf{G P a})\end{array}$ & $\mathbf{M}_{\text {pic }}$ & $\boldsymbol{\phi}\left({ }^{\circ}\right)$ & $\begin{array}{c}\mathbf{R}_{\mathbf{c}} \leftrightarrow \mathbf{f}_{\mathbf{b}} \\
(\mathbf{M P a})\end{array}$ & $\begin{array}{c}\mathbf{R}_{\mathbf{t}} \leftrightarrow \mathbf{f}_{\mathbf{t}} \\
(\mathbf{M P a})\end{array}$ \\
\hline 15,8 & 2,83 & 74 & 11,55 & 0,61 \\
\hline
\end{tabular}

\subsection{Classement de la Grave routière à base de MIDND Traitée au Liant Hydraulique}

Le classement d'une Grave Traitée au Liant Hydraulique (GTLH) se fait en fonction des valeurs du module d'Young et de la résistance en traction. Le matériau a été classé en catégorie mécanique 3 (cf. figure 11).

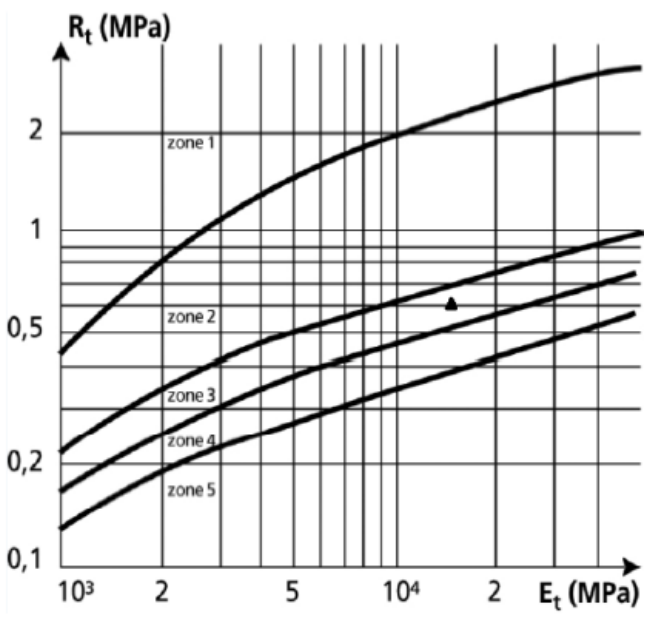

Fig.11. Classement de la Grave routière à base de MIDND Traitée au Liant Hydraulique

\section{Discussions et conclusions}

Les premiers résultats d'indentation sur particule isolée de mâchefer montrent une faible rigidité homogénéisée.
Une étude statistique serait intéressante pour avoir une idée plus précise de l'ordre de grandeur du module élastique de ces types de matériaux du moment que leurs zones de production sont diverses. La modélisation développée a permis de prédire le comportement des mâchefers lorsqu'ils sont traités au ciment. L'angle de frottement interne est comparable à celui déterminé dans les expérimentations de [7] et à celui des graves routières [8]. Les valeurs obtenues s'expliquent par le fait que le matériau granulaire est composé de grains anguleux bien gradués. Le classement mécanique de la grave routière à base de MIDND traitée au ciment en catégorie 3 prouve la faisabilité d'un réemploi de ce type de Sous-Produits Industriels dans ce secteur d'activités.

\section{Remerciements}

Les auteurs remercient MM. Gérard Mauvoisin et Olivier Bartier pour leur aide et leur expertise dans la campagne expérimentale de tests d'indentation, ainsi que l'entreprise Eurovia, en particulier M. Loïc Seguin, pour leur approvisionnement en MIDND.

\section{Références}

1. Arrêté relatif au recyclage en technique routière des mâchefers d'incinération de déchets non dangereux. Ministère de l'écologie, du développement durable, des transports et du logement, France (18 Novembre 2011).

2. W. C. Oliver, G. M. Pharr, An improved technique for determining hardness and elastic modulus using load and displacement sensing indentation experiments. J. Mater. Res. 7 (1992) 1564-1583.

3. L. Sow, S. Kamali-Bernard, O. Bartier, G. Mauvoisin, F. Bernard, Experimental estimation of the elastic modulus of non-hazardous waste incineration bottom ash aggregates by indentation tests - Microanalysis of particles by scanning electron microscopy. Submitted in Adv. Mater. Res. (Trans Tech Publications).

4. J. Lee, G. L. Fenves, Plastic-damage model for cyclic loading of concrete structures. J Eng. Mech., ASCE, 124 (1998) 892-900.

5. R. Javier, M. Francisco, and M. Joaquín, Concrete Constitutive Model, Calibration and Applications. Simulia Community Conference (2013).

6. F. Bernard and S. Kamali-Bernard, Predicting the evolution of mechanical and diffusivity properties of cement pastes and mortars for various hydration degrees - A numerical simulation investigation. Comp. Mat. Sci. 61 (2012) 106-115.

7. F. Becquart, Première approche du comportement mécanique d'un milieu granulaire issu d'un mâchefer d'incinération d'ordures ménagères : valorisation en technique routière. $\mathrm{PhD}$ thesis. Université des sciences et technologies de Lille (2007).

8. P. Hornych, A. Kazai, Q. Quibel, Study of the resilient behaviour of unbound granular materials, Proc. 5th Conference on Bearing Capacity of Roads and Airfields. Trondheim, Norway, 3 (1998) 12771287. 